\title{
The Transition Temperature of the Nuclear Caloric Curve
}

\author{
A. Barrañón ${ }^{1}$, J. Escamilla Roa ${ }^{2}$, and J. A. López ${ }^{2}$ \\ ${ }^{1}$ Universidad Autónoma Metropolitana - Azcapotzalco, México D.F., México \\ ${ }^{2}$ Department of Physics, University of Texas at El Paso, El Paso, Texas 79968, U.S.A.
}

Received on 11 September, 2003

\begin{abstract}
Experimental studies have obtained the caloric curve of nuclear matter from heavy ion collisions as well as its dependence on the size of the fragmenting source. In particular it has been determined that smaller systems have caloric curves with higher plateau temperatures than larger systems. This work uses molecular dynamics simulations to study the thermodynamics of heavy ion collisions and to identify the main factors that determine the caloric curve. The simulations indicate that the reaction is composed of three stages: 1) an initial collision that transforms the nuclei from normal density and zero temperature and entropy, to a hot and dense blob of matter with higher values of density, temperature and entropy; 2) this is followed by a constant-entropy expansion that takes the system to the spinodal of the phase diagram; 3 ) where the system rapidly disassembles into fragments by the process of spinodal decomposition, and not by nucleation. These findings indicate that the plateau temperature of the caloric curve is nothing more than the temperature of the phase change and it is set by the intersection of the isentropic expansion and the spinodal. In other words, the plateau temperature is simply the temperature at which the system breaks as it enters the spinodal. This transition temperature is thus set by the entropy generated during the initial part of the collision.
\end{abstract}

\section{Introduction}

The caloric curve $(C C)$ of nuclear matter, i.e. the relation between the system's temperature and its excitation energy at fragmentation time, has been studied experimentally and theoretically for almost a decade now [1-10]. Recently, these studies have turned into an analysis of the size dependence of the $C C$ [11].

Combining data from several experimental measurements, the colleagues from the Texas A\&M University found various values of the plateau temperature, $T_{p}$, of $C C$ 's obtained for systems of different sizes [11]. Caloric curves from systems with masses between 30 to 60 nucleons have a $T_{p} \approx 9 \mathrm{Mev}$, while systems with masses between 180 to 240 yield a $T_{p} \approx 6 \mathrm{MeV}$. In explaining this effect, the study draws a similarity between these temperatures and the Coulomb instability limiting temperatures [12], although molecular dynamics simulations of disassembly of excited Lennard-Jones drops show that Coulomb does not play a deciding role in the breakup [13].

Using theoretical analysis and molecular dynamics simulations, a more recent study [14] investigated the question of what really determines the transition temperature in nuclear reactions. This article submits more supporting evidence to the premise advanced in that previous study.

\section{The transition temperature $T_{p}$}

Following the arguments presented a decade ago by Bertsch and Siemens $[15,16]$, now supported by molecular dynamics calculations [17], a heavy ion collision can be visualized as a collision in which the originally cold nuclei fuse into a blob of nucleons that reaches some maximum density and temperature. In the density-temperature phase diagram of the equation of state of, for instance, $[18,19]$ this can be represented as a displacement from normal saturation density and zero temperature.

This initial compression is followed by an isentropic expansion that cools and reduces the density of the blob. This expansion drives the system into lower density and cooler temperatures until the system enters the spinodal line where mechanical instabilities grow breaking the system by spinodal decomposition. This breakup is identifiable by the existence of an imaginary speed of sound. This scenario, for an entropy of $S=1.5$, can be represented in the phase diagram of nuclear matter as shown in Fig. 1.

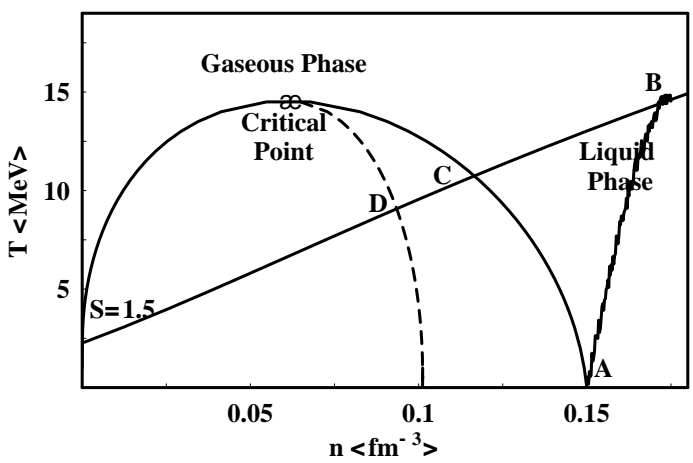

Figure 1. Possible trajectory of a heavy ion collision plotted on the phase diagram of reference [18]. The solid semi-circle represents the boundary of the phase coexistence region, the dashed line is the spinodal line, and the traverse line represents the trajectory of a $S=1.5$ isentropic expansion. 
According to this scenario, the breakup occurs when the system, expanding along a constant entropy curve reaches the spinodal. As this disassembly into clusters occurs very fast, the breakup temperature, i.e. the plateau temperature of the $C C$, is the temperature the system has when it reaches the spinodal. In other words, the $T_{p}$ of a reaction is determined by the entropy generated in the initial stage of the collision, and not necessarily by the size of the compound nucleus.

\subsection{Molecular dynamics simulations}

As the initial generation of entropy occurs out of equilibrium, we study it using the molecular dynamics $(M D)$ code "LATINO" [17]. This model combines $M D$ with a fragment-recognition algorithm, that has been applied successfully to study, among other things, phase transitions [20] and critical phenomena [21] in nuclear reactions. LATINO uses a potential [22] that reproduces nucleon cross sections and the binding energies and densities of real nuclei, along with a Coulomb term. Using this $M D$ code, central collisions were performed for several reactions. In this study we present results for the reaction $N i+N i$ at beam energies of $E=2251 \mathrm{MeV}$; other aspects of these simulations have been published elsewhere [14,21,23]. Fig. 2 shows a the caloric curve obtained from hundreds of $N i+N i$ reactions at varying energies.

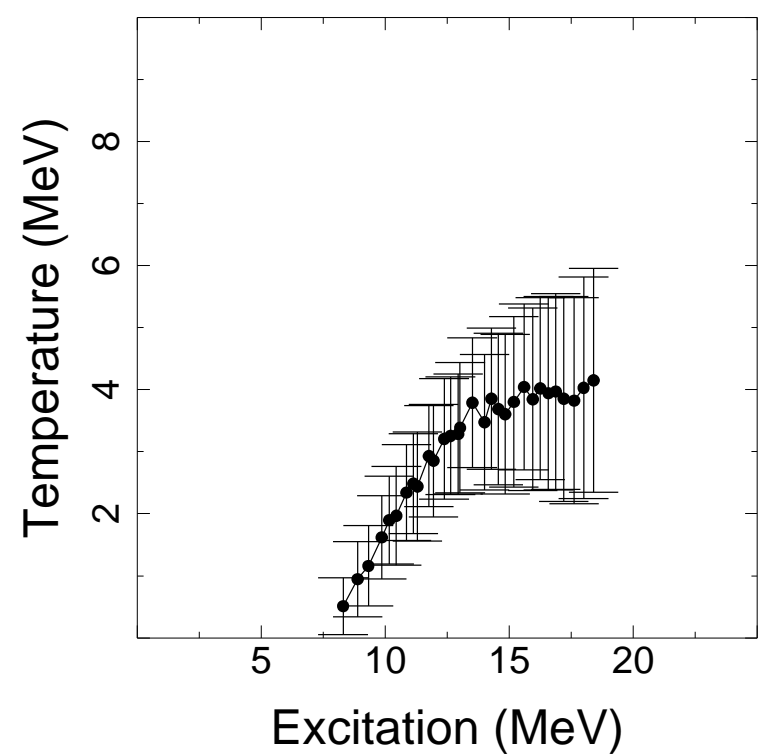

Figure 2. Caloric curve obtained for the reaction $N i+N i$ at varying energies.

During the collision, the density and temperature of the $50 \%$ most central particles was determined as a function of time, and this information was then used to study the generation of entropy. To track the entropy generated at the "quasiclassical" hot and dense stage of the reaction we estimate it as if dealing with a classical gas [24], namely

$$
S=\log \left[\frac{1}{n}\left(\frac{3 T}{2}\right)^{3 / 2}\right]+S_{o},
$$

where $n$ is the particle density, $S$ the entropy per particle in units of Boltzmann's constant, and $S_{o}$ is a constant that depends on the nucleon mass. Fig. 3 shows the time evolution of the square of the speed of sound, temperature, density and entropy of one reaction of $N i+N i$ at $2251 \mathrm{MeV}$. The square of the adiabatic speed of sound is obtained using

$$
v_{s}^{2}=\frac{1}{m}\left[\frac{10}{9}<\epsilon_{k}>+a \frac{n}{n_{o}}\right]_{S}+b \sigma\left(\frac{n}{n_{o}}\right)^{\sigma},
$$

with $\epsilon_{k}$ denoting the kinetic energy per nucleon, $\rho$ the mass density, $a=-358.1 \mathrm{MeV}, b=304.8 \mathrm{MeV}$ and $\sigma=7 / 6$.

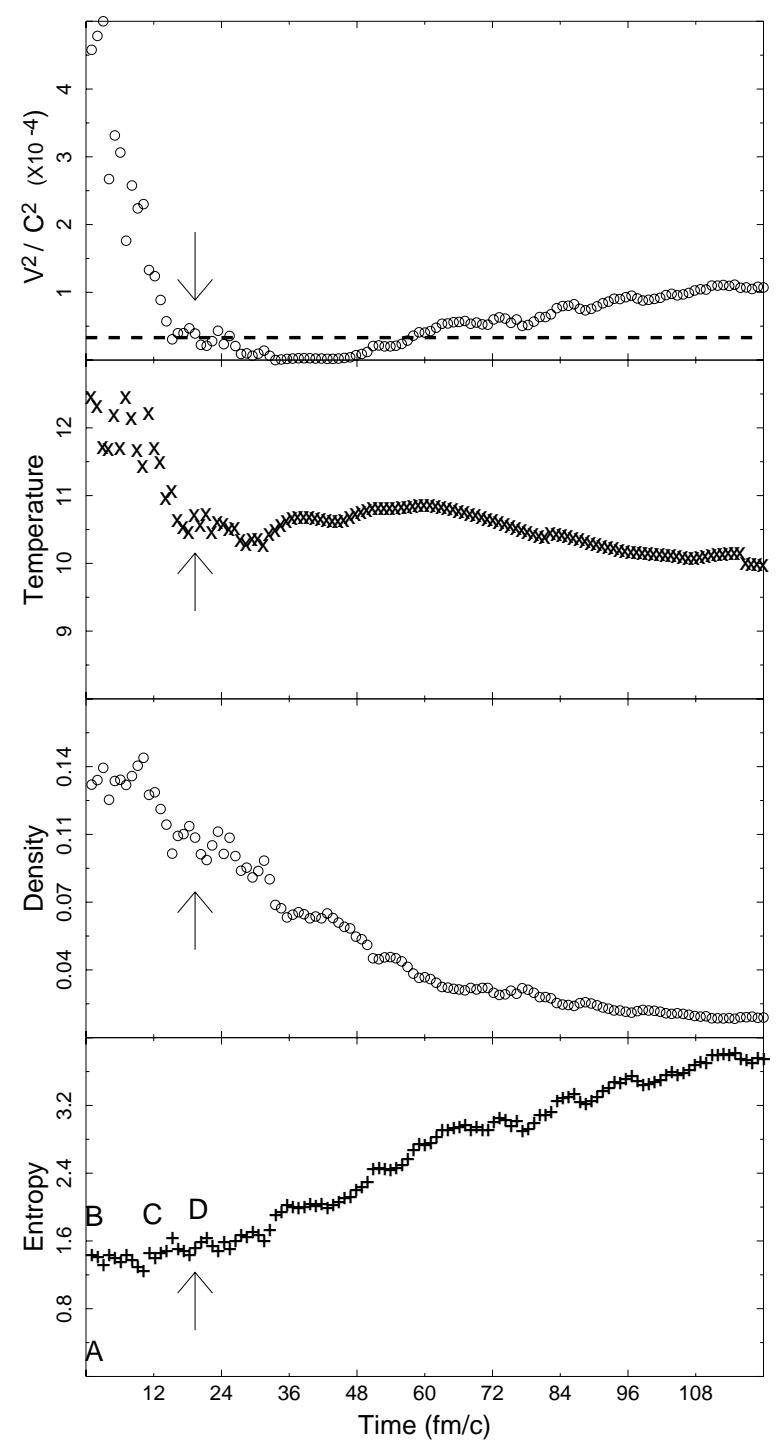

Figure 3. Time evolution of the speed of sound squared, $v_{s}^{2}$ (in units of $c^{2}$ ), temperature (in $\mathrm{MeV}$ ), nucleon density $\left(\mathrm{fm}^{-3}\right)$, and entropy per particle of a central collision of $N i+N i$ at $2251 \mathrm{MeV}$.

The scenario of section 2 is apparent from the bottom panel. Starting from zero entropy (near point $\mathbf{A}$ ), the compound nucleus generates entropy and reaches a maximum 
rapidly at $(\mathbf{B})$, expanding then with a constant value of $S$, entering the coexistence region (around $\mathbf{C}$ ), and continuing until the spinodal (D) where $S$ increases due to the phase change. The time when $v_{s}^{2}$ becomes negative (as marked by the arrows), indicates when the nucleus reaches the spinodal.

Since it is only an expression for a classical gas, equation (1) does not yield correct numerical values for $S$. To correct this, we use the values of $n$ and $T$ at the time when $v_{s}^{2}$ becomes negative, and use them to determine the value that the entropy should have at that point. $S_{o}$ is then set to the value needed to have equation (1) yield the appropriate value of $S$ at point $\mathbf{D}$ (see [14] for details).

\section{Conclusions}

The $M D$ simulations presented provide further support to the scenario collision - isentropic expansion - spinodal decomposition put forward in [14]. The main results of this study is that the entropy produced in the initial stage of the reaction defines the trajectory that is followed by the compound nucleus in its expansion into the spinodal line where it disassembles. Therefore, the transition temperature $T_{p}$ is defined by the intersection of the isentrope and the spinodal. The remaining question to be answered is what is the exact role of the system size in producing more or less entropy.

\section{Acknowledgments}

A.B. and J.A.L. acknowledge the hospitality of the Universidad of Colima, in Colima, Mexico.

\section{References}

[1] J. Pochodzalla et al., Prog. Part. Nucl. Phys 39, 43 (1997).

[2] H. Feldmeier et al., Gross properties of nuclei and nuclear excitations GSI, Darmstadt, (1999).

[3] B.-A. Li and W. U.Schröder, Eds., Isospin physics in heavy ion collisions at intermediate energies Nova Science Publishers, Inc., New York, (2001).
[4] J. B.Natowitz et al., Phys. Rev. C52, R2322 (1995).

[5] X. Campi, H. Krivine, and E. Plagnol, Phys. Lett.B 385, 1 (1996).

[6] V. Viola, et al., Phys. Rev. C59, 2660 (1999).

[7] J. Pochodzalla et al., Phys. Rev. Lett. 75, 1040 (1995).

[8] V. Serfling et al., Phys. Rev. Lett. 80, 3928 (1998).

[9] J. A. Hauger et al., Phys. Rev. Lett. 77, 235 (1996).

[10] G. Papp and W. Nörenberg, Heavy Ion Phys. 1, 241 (1995).

[11] J. B. Natowitz et al., Phys. Rev. C65, 034618 (2002).

[12] L. L. Zhang, H. Q. Song, P. Wang and R. K. Su, Phys. Rev. C59, 3292 (1999).

[13] P. Balenzuela and C. O. Dorso, Phys. Rev. C65, 057602 (2002).

[14] A. Barrañón, J. Escamilla Roa and J. A. López, Phys. Rev. C, 69, 014601 (2004)

[15] G. F. Bertsch, and P. Siemens, Phys. Lett. B126, 9 (1983).

[16] J. A. López, and P. Siemens, Nucl. Phys A314, 465 (1984).

[17] A. Barrañón, A. Chernomoretz, C. O. Dorso, J. A. López, and J. Morales, Rev. Mex. Phys. 45-Sup.2, 110 (1999).

[18] J. A. López and C. O. Dorso, Lecture Notes on Phase Transitions in Nuclear Matter World Scientific, Singapore, (2000).

[19] J. Escamilla and J. A. López, (to be published).

[20] A. Barrañón, C. O. Dorso, and J. A. López, Rev. Mex. Phys. 47-Sup.2, 93 (2001).

[21] A. Barrañón, R. Cárdenas, C. O. Dorso, and J. A. López, Heavy Ion Phys. 17-1, 59 (2003).

[22] R. J. Lenk, T. J. Schlagel and V. R. Pandharipande, Phys. Rev. C42, 372 (1990).

[23] A. Barrañón, C. O. Dorso and J. A. López, Información Tecnológica (ISSN: 0716-8756) 14, 31 (2003).

[24] K. Huang, Statistical Mechanics, 2nd edition John WIley \& Sons, New York, (1987). 